malignancy at the time of the CT scan compared to patients with known malignancy $(69.4 \%$ vs $46.0 \%, \mathrm{p}=0.0046)$, or if metastases were present at the time of CT scan $(58.3 \%$ vs $26.7 \%$, $\mathrm{p}=0.0012)$.

There were $86(53.8 \%)$ central (main or lobar pulmonary arteries), 60 (37.5\%) segmental, and 14 (8.8\%) subsegmental pulmonary emboli. No significant mortality difference was observed between these radiological features.

Conclusion This study has assessed potential poor prognostic features in patients with cancer and iPE. Despite the vast majority receiving therapeutic anticoagulation, there is a high 30-day and 6-month mortality. The benefits of conventional treatment in this clinical situation are as yet unclear.

\section{P168 REDUCED GAS TRANSFER (TLCO) PREDICTS POOR OUTCOME IN PATIENTS WITH PULMONARY HYPERTENSION AND HEART FAILURE WITH PRESERVED EJECTION FRACTION}

N Hussain, S Ramjug, C Billings, J Hurdman, CA Elliot, R Condliffe, DG Kiely. Sheffield Pulmonary Vascular Disease Unit, Sheffield, UK

\subsection{6/thoraxjnl-2014-206260.297}

Rationale There is limited data on predictors of survival in patients with Pulmonary Hypertension $(\mathrm{PH})$ in the context of Heart Failure and Preserved Ejection Fraction (HF-pEF). Simple non-invasive tests to aid the physician in prognostication would be valuable. The aim of this study was to examine demographic and non-invasive predictors of outcome in PH-HF-pEF in a large well phenotyped PH registry.

Method In the ASPIRE Registry (Hurdman J et al Eur Resp J, 2012), 1737 consecutive, incident, treatment-nave patients with suspected PH underwent diagnostic evaluation between February 2001 and 2010. Patients were diagnosed as PH-HF-pEF if no other causes of PH could be identified and they fulfilled the following criteria: signs and symptoms of heart failure; mean pulmonary artery pressure $\geq 25 \mathrm{mmHg}$ at rest and pulmonary arterial wedge pressure $>15 \mathrm{mmHg}$ by RHC; preserved left ventricular systolic function (ejection fraction $\geq 50 \%$ ) by echocardiography or CMR. Predictors of survival were assessed using forward stepwise Cox regression analysis. Variables with a p-value

Results 98 patients who fulfilled the diagnostic criteria for PH-HF-pEF were identified. Maximum duration of follow-up was 10 years with a mean follow up $4.9 \pm 2.3$ years, during which 33 (34\%) patients died. After multivariate analysis, only ISWT distance HR 0.99 CI (0.99-1.00) and TLCO HR 0.96 CI(0.94-0.98) at baseline, were predictors of outcome $(\mathrm{p}<0.01)$. Median predicted TLCO in the PH-HF-pEF population was $65 \%$. The 5 -year survival in those with a TLCO $<65 \%$ predicted was $60 \%$, compared with $85 \%$ in those whose TLCO was $\geq 65 \%$ ( $p<0.01$ ).

Conclusions Simple non-invasive testing such as TLCO and exercise capacity measured by the ISWT predict outcome in patients with PH-HF-pEF.

\section{P169 RATES OF RECOVERY OF OXYGEN CONSUMPTION AND HEART RATE AFTER CARDIOPULMONARY EXERCISE TESTING PREDICT SURVIVAL IN PATIENTS WITH PRECAPILLARY PULMONARY HYPERTENSION}

SD Thomson, AJ Peacock, MK Johnson. Scottish Pulmonary Vascular Unit, Glasgow, Scotland, UK

10.1136/thoraxjnl-2014-206260.298

\begin{tabular}{|c|c|c|c|}
\hline Variable & Significance level & Variable & Significance level \\
\hline V02 R 30 & 0.729 & OUES & 0.026 \\
\hline HRR $30^{*}$ & 0.300 & Peak V02 & 0.024 \\
\hline V02 R 60 & 0.084 & VE/VCO2 at AT & 0.032 \\
\hline HRR 60 & 0.058 & Peak heart rate & 0.067 \\
\hline V02 120 & 0.021 & Diagnosis & 0.055 \\
\hline HRR 120 & 0.003 & Age & $<0.001$ \\
\hline DLCO (\% pred) & 0.002 & $\operatorname{logNTproBNP}$ & 0.064 \\
\hline SvO2 & 0.115 & Cardiac index & 0.278 \\
\hline RAP & 0.401 & mPAP & 0.532 \\
\hline 6MWD & 0.205 & WHO FC & 0.428 \\
\hline
\end{tabular}

VO2 R 30,60, $120-\mathrm{VO}_{2}$ at $30,60,120$ seconds of recovery as a percentage of peak $\mathrm{VO}_{2}$ : HRR 30,60, 120 - Heart rate recovery at 30,60,120 seconds of recovery as a percentage of peak heart rate; DLCO - diffusion capacity of the lungs for carbon monoxide as a percentage of predicted value; SvO2 - resting mixed venous oxygen saturation; RAP - resting right atrial pressure; 6MWD - 6 min walk distance; OUES - oxygen uptake efficiency slope; mPAP - resting mean pulmonary arterial pressure; WHO FC - WHO functional class, I/II v III/IV

Introduction Several cardiopulmonary exercise testing (CPET) variables have been shown to predict prognosis in pulmonary hypertension (PH). ${ }^{1}$ Recently published data suggests that novel variables such as oxygen uptake efficiency slope (OUES), i.e. the relationship between $\mathrm{VO}_{2}$ and log-transformed ventilation ${ }^{2}$ and heart rate recovery (HRR), the rate of decline of heart rate at one minute after an incremental CPET, have been shown to predict survival in a cohort of $\mathrm{PH}$ patients.

We aimed to study the prognostic significance of the rate of recovery of $\mathrm{VO}_{2}$ after incremental CPET alongside HRR and OUES in a large cohort of patients with precapillary $\mathrm{PH}$. We hypothesised that a slower $\mathrm{VO}_{2}$ recovery would be associated with poorer survival and that we could confirm that lower HRR and OUES are significantly associated with a worse outcome.

Method Retrospective analysis was undertaken of data from108 incident patients who underwent CPET at the time of diagnosis of Group I or IV PH. Univariate Cox proportional hazard analyses were undertaken to assess the prognostic significance of the variables considered and the results are shown in Table 1.

Results [Table 1]

Conclusions The degree of $\mathrm{VO}_{2}$ recovery at 120 seconds after incremental CPET is predictive of survival in this relatively large group of patients with precapillary $\mathrm{PH}$. We have also confirmed the findings seen in another centre of a significant influence of heart rate recovery and OUES on survival. Further work should focus on whether these variables provide additional prognostic information over their more traditionally studied counterparts.

\section{REFERENCES}

1 Johnson MJ, Thomson SD. The role of exercise testing in the modern management of pulmonary arterial hypertension Diseases 2014;2(2):120-47

2 Ramos, et al. Exercise oxygen uptake efficiency slope independently predicts poor outcome in pulmonary arterial hypertension. Eur Respir J 2014;43(5):1510-12

\section{P170 HEART RATE RECOVERY AT ONE MINUTE FOLLOWING INCREMENTAL SHUTTLE WALK TEST PREDICTS OUTCOME IN PULMONARY HYPERTENSION}

CG Billings, J Hurdman, M Austin, I Armstrong, CA Elliot, RA Condliffe, DG Kiely. Sheffield Pulmonary Vascular Disease Unit, Sheffield, UK

10.1136/thoraxjnl-2014-206260.299

Background Heart-rate recovery during the first minute of rest (HRR1) after a six minute walk test (6MWT) has been shown to 